\title{
Caracterização socioeconômica e condição de saúde oral de idosos atendidos em uma clínica-escola de odontologia
}

\section{Socioeconomic characterization and oral health condition of the elderly served in a clinical dental school}

\section{Caracterización socioeconómica y condición de salud oral de ancianos atendidos en una clínica-escuela de odontología}

\section{Recebido: 10/04/2016 \\ Aprovado: 18/08/2016 \\ Publicado: 01/09/2016}

\section{Isabella Lima Arrais Ribeiro ${ }^{1}$ Eduardo Dias Ribeiro ${ }^{2}$ Waldemir Gonçalves de Abrantes ${ }^{3}$ Celso Koogi Sonoda ${ }^{4}$}

Este estudo tem como objetivo caracterizar socioeconomicamente e identificar as condições de saúde oral e os hábitos de pacientes idosos atendidos em uma clínica-escola de Odontologia. Realizou-se um estudo quantitativo com dados secundários de 126 prontuários de pacientes idosos na clínica-escola do Centro Universitário de João Pessoa-PB. A média de idade foi de 67,3 anos; com 72,2\% do gênero feminino; $61,1 \%$ brancos; $43,7 \%$ eram tabagistas; $28,6 \%$ etilistas e 0,8\% usuário de drogas. Observou-se que 49,2\% dos prontuários tinham registro de lesão/alteração na mucosa oral. 0 uso de prótese dentária foi verificado em $54,8 \%$ e a necessidade do uso em 65,9\%. Esse estudo revelou as condições de saúde, bem como as necessidades de cuidado requeridas pelos idosos que procuram o serviço de Odontologia, sendo necessário olhar a essa demanda, bem como às peculiaridades que envolvem o atendimento ao idoso, no intuito de atendêlo de forma integral, visando proporcionar um aumento na qualidade de vida.

Descritores: Saúde bucal; Idoso; Mucosa bucal.

This study aim to characterize socioeconomically and identify the conditions of health oral and habits of elderly patients attended in a clinical dental school of Odontology. Was performed a study in 126 records of elderly patients in clinical dental school of University Center of João Pessoa-PB, Brazil. The mean age was 67.3 years, with $72.2 \%$ female, $61.1 \%$ white. $43.7 \%$ were smokers; $28.6 \%$ consumed alcohol and $0.8 \%$ drug user. It was observed that $49.2 \%$ of records had lesion/alteration in the oral mucosa. The use of dental prosthesis was observed in $54.8 \%$ and the need to use in $65.9 \%$. This study revealed the health conditions, as well as the needs of care required by the elderly looking for Dentistry service, and must look to this demand, as well as the peculiarities involving elderly care, in order to serve him fully, in order to provide an increase in the quality of life.

Descriptors: Oral health; Aged; Mouth mucosa.

Este estudio tiene como objetivo caracterizar socioeconómicamente e identificar las condiciones de salud oral y los hábitos de los pacientes ancianos tratados en una clínica universitaria de Odontología. Se realizó un estudio cuantitativo con datos secundarios de 126 historias clínicas de ancianos de la clínica-escuela del Centro Universitario de João Pessoa-PB, Brasil. La edad promedio fue de 67,3 años, con el 72,2\% de mujeres, $61,1 \%$ de blancos, $43,7 \%$ eran fumadores, $28,6 \%$ usuarios de alcohol y 0,8\% de drogas. Se observó que el 49,2\% de los registros médicos mostró lesión / cambio en la mucosa oral. Se observó que el uso de prótesis dentales en un $54,8 \%$ y la necesidad de utilizar en un $65,9 \%$. Este estudio reveló las condiciones de salud, así como las necesidades de cuidado requeridas por los ancianos que buscan el servicio de Odontología siendo necesario prestar atención a esta demanda, así como las particularidades que implican el cuidado de los ancianos, para brindarles un servicio completo, con el objetivo de proporcionarles un aumento en la calidad de vida.

Descriptores: Salud bucal; Anciano; Mucosa bucal.

1 Cirurgião-Dentista. Especialista em Endodontia. Mestre em Diagnóstico Bucal. Doutora em Modelos de Decisão e Saúde. PósDoutora em Epidemiologia. isabella_arrais@yahoo.com. Brasil.

2 Cirurgião Dentista e Buco-Máxilo-Facial. Especialista em Estomatologia. Mestre em Estomatologia. Doutor em Cirurgia e Traumatologia Buco-Maxilo-Facial. Professor Adjunto da Universidade Federal de Campina Grande/PB. eduardodonto@yahoo.com.br. Brasil.

${ }^{3}$ Cirurgião-Dentista. Especialista em Saúde Pública. Especialista em Cirurgia Oral Menor. Especializando em Ortodontia pela Faculdade de Tecnologia de Sete Lagoas. waldemirbrantes@hotmail.com.com. Brasil.

${ }^{4}$ Cirurgião Dentista. Mestre e Doutor em Cirurgia e Traumatologia Buco-Maxilo-Facial. Professor Adjunto do Departamento de Cirurgia e Clínica Integrada da Faculdade de Odontologia de Araçatuba - Universidade Estadual Paulista - UNESP, Araçatuba/SP, Brasil. sonohara@terra.com.br. Brasil. 


\section{INTRODUÇÃO}

$\mathrm{A}$ s transformações que ocorrem na sociedade contemporânea direcionam para o aumento na expectativa de vida da população e estima-se que as mudanças que corroboram para esse fato estejam relacionadas às conquistas na área da medicina e à amplitude das políticas de atenção à saúde do idoso ${ }^{1,2}$. O Relatório de Desenvolvimento Humano 2014 divulgado pelo Programa das Nações Unidas para o Desenvolvimento (PNUD) mostra que a expectativa de vida no Brasil aumentou 17,9\% entre 1980 e 2013, passando de 62,7 para 73,9 anos, com um aumento real de 11,2 anos e estima-se que em 2020, a população com mais de 60 anos no país deve chegar a 30 milhões de pessoas, ou seja, $13 \%$ do total 2 .

$\mathrm{Na}$ Odontologia, o atendimento ao indivíduo idoso requer uma avaliação prévia do histórico de saúde oral, com o levantamento de informações sobre tratamentos anteriores e avaliação das necessidades de reabilitação, com vista a melhorar a autoestima e a qualidade de vida. Além disso, é fundamental que se tenha o conhecimento das alterações sistêmicas, bem como dos hábitos adquiridos pelo paciente idoso. Todos os fatores somados (histórico de saúde oral, sistêmico e hábitos) fornecem informações importantes para o prognóstico de um possível tratamento a ser realizado, bem como orienta para a causa de lesões e/ou alterações que podem ser observadas com mais frequência para essa faixa etária ${ }^{3}$.

Esse estudo teve como objetivo caracterizar socioeconomicamente e identificar as condições de saúde oral e os hábitos de pacientes idosos atendidos na clínica-escola de Odontologia do Centro Universitário de João Pessoa-PB.

\section{MÉTODO}

O presente estudo tem a aprovação do Comitê de Ética em Pesquisa com Seres Humanos sob o parecer de número 194.077 de 06/02/2013 e caracteriza-se por ser uma pesquisa de campo aplicada, quantitativa, com procedimentos exploratórios descritivos e inferenciais, adotando-se uma abordagem indutiva com técnica de observação indireta 4 .

Esse estudo foi realizado na Clínica Escola de Estomatologia do Curso de Odontologia do Centro Universitário de João Pessoa-PB (UNIPÊ) e teve como universo da pesquisa todos os prontuários de pacientes atendidos na referida clínica no período de agosto de 2009 a maio de 2012 sendo selecionados para compor a amostra os prontuários de pacientes com idade acima dos 60 anos, de ambos os gêneros, sendo excluídos do universo da pesquisa prontuários de crianças, adolescentes e pacientes abaixo de 60 anos, além dos que tinham ausência das informações de interesse, ficando a amostra composta por 126 prontuários de pacientes que atenderam aos critérios de inclusão no referido período.

A coleta de dados foi realizada mediante análise dos prontuários e preenchimento de fichas clínicas confeccionadas para o registro das seguintes variáveis de interesse: dados socioeconômicos e demográficos (gênero, idade, etnia, renda familiar, cidade de origem); dados relativos aos hábitos (tabagismo, etilismo e uso de drogas), bem como o índice CPOD (número de dentes Cariados, Perdidos e Obturados na dentição permanente), lesões e/ou alterações na mucosa oral e uso/necessidade de prótese dentária.

Os dados foram inseridos em uma planilha do Excel e analisados pelos softwares IBM SPSS (Versão 20.0) e R (Versão 2.15.1), sendo realizadas as seguintes análises: valores de frequência e percentuais para cada variável e suas categorias e testes Qui-Quadrado ou Exato de Fisher para avaliar a existência de dependência entre os diferentes gêneros (Masculino e Feminino) e as variáveis de interesse, bem como, para avaliar a associação entre o uso de prótese dentária e a presença de lesões e/ou alterações na mucosa oral. Para a análise das variáveis quantitativas contínuas, como idade e índice CPOD, realizou-se análise de normalidade na distribuição dos dados, mediante o teste de Kolmogorov-Smirnov, 
verificando-se que, para ambas as variáveis, a distribuição dos valores foi do tipo "nãonormal" ( $p=0,000)$, sendo realizado o teste de Mann-Whitney para a verificação da diferença entre as médias de idade e de índice CPOD entre os gêneros e entre os pacientes que tinham ou não lesão e/ou alteração na mucosa oral. Avaliou-se ainda pelo teste de KruskalWallis se houve diferença na distribuição do CPOD entre as idades dos pacientes. Para todos os testes realizados adotou-se um nível de significância de 5\%.

\section{RESULTADOS}

Dos 126 prontuários avaliados, observou-se que a idade média dos pacientes foi de 67,3 $( \pm 6,2)$, com mínimo de 60 e máximo de 86 anos, sendo $35(27,8 \%)$ do gênero masculino e 91 (72,2\%) do feminino, com 77 (61,1\%) declarados brancos e 49 (38,9\%) não-brancos, $81(64,3 \%)$ apresentando renda mensal de até 2 salários mínimos (SM's) e 45 (35,7\%) com renda de 3 ou mais SM's, sendo 101 (80,2\%) residentes na Paraíba e 25 (19,8\%) residentes em outro Estado.

Quanto aos hábitos, $55 \quad(43,7 \%)$ pacientes eram tabagistas; $36(28,6 \%)$ etilistas e $1(0,8 \%)$ usuário de drogas.

Dos 126 pacientes, observou-se que 62 $(49,2 \%)$ tinham algum registro de lesão/alteração na mucosa oral, sendo: 26 $(20,6 \%)$ compatíveis com varicosidades linguais, $14(11,1 \%)$ com estomatite protética e $7(5,6 \%)$ com hiperplasia fibrosa inflamatória e $15(11,95)$ com outros tipos não especificados.

0 uso de prótese dentária foi verificado em $69(54,8 \%)$ dos pacientes e a necessidade do uso em $83(65,9 \%)$.

0 CPOD médio dos pacientes foi de 26,0 $( \pm 5,6)$, com mínimo de 9 e máximo de 32 .

A média de idade para o gênero masculino foi de $67,14( \pm 5,8)$ anos e para o feminino foi de $67,43( \pm 6,34)$, não sendo verificadas diferenças estatisticamente significantes $(p=0,930)$; bem como para o
CPOD $(\mathrm{p}=0,326)$, sendo a média do índice de $25,43( \pm 5,6)$ para o gênero masculino e de $26,33( \pm 5,6)$ para o feminino.

No entanto, foi verificada diferença estatisticamente significante entre o CPOD apresentado pelos pacientes de diferentes idades $(p=0,029)$, sendo verificada essa diferença para as idades entre 60 e 67 anos, que obtiveram os maiores valores, comparado às demais idades.

A tabela 1 mostra a distribuição dos pacientes por gênero para as variáveis em estudo. Já a tabela 2 mostra a distribuição dos pacientes segundo o registro de lesões na mucosa oral frente às variáveis de interesse.

\section{DISCUSSÃO}

O Centro Universitário de João Pessoa conta com uma clínica-escola de Odontologia que atende gratuitamente pacientes de todas as idades. Esses pacientes adquirem um vínculo com a instituição, mediante inscrição com abertura e arquivamento de prontuário, tendo o seu tratamento garantido, desde a triagem, na clínica da disciplina de Estomatologia, até o encerramento do tratamento, com o direcionamento às demais clínicas que se fizerem necessárias.

O presente estudo possui a limitação de não poder estender os seus resultados a toda população de idosos da região, em razão de não ter uma amostra probabilística que a represente. Dessa forma, os dados obtidos podem caracterizar socioeconomicamente apenas e quanto às condições de saúde bucal os idosos que foram atendidos na referida clínica-escola no período agosto de 2009 a maio de 2012.

Assim como em outras pesquisas epidemiológicos similares, a procura pelo atendimento odontológico no presente estudo foi maior pelo gênero feminino ${ }^{5-9}$ bem como, por pessoas declaradas com cor de pele branca $^{5,8}$ e renda superior a 1 salário mínimo ${ }^{7}$. 
Tabela 1. Distribuição (frequência e valores percentuais) por gênero dos pacientes quanto às variáveis de interesse de 2009 a 2012. João Pessoa, PB, 2014

\begin{tabular}{|c|c|c|c|c|c|c|}
\hline \multirow[t]{3}{*}{ Variáveis } & \multirow[t]{3}{*}{ Categorias } & \multicolumn{3}{|c|}{ Gênero } & & \multirow[t]{3}{*}{ Significância } \\
\hline & & Masculino & & Feminino & & \\
\hline & & $n=35$ & $\%$ & $n=91$ & $\%$ & \\
\hline \multirow[t]{2}{*}{ Etnia } & Branco & 35 & $100,0 \%$ & 42 & $46,2 \%$ & $0,000^{*}$ \\
\hline & Não-Branco & 0 & $0,0 \%$ & 49 & $53,8 \%$ & \\
\hline \multirow[t]{2}{*}{ Renda familiar } & Até 2 SM' & 35 & $100,0 \%$ & 46 & $50,5 \%$ & $0,000^{*}$ \\
\hline & 3 ou mais SM's & 0 & $0,0 \%$ & 45 & $49,5 \%$ & \\
\hline \multirow{2}{*}{$\begin{array}{l}\text { Cidade de } \\
\text { origem }\end{array}$} & Paraíba & 35 & $100,0 \%$ & 66 & $72,5 \%$ & $0,000^{*}$ \\
\hline & Outro Estado & 0 & $0,0 \%$ & 25 & $27,5 \%$ & \\
\hline \multirow[t]{2}{*}{ Tabagismo } & Sim & 35 & $100,0 \%$ & 20 & $22,0 \%$ & $0,000^{*}$ \\
\hline & Não & 0 & $0,0 \%$ & 71 & $78,0 \%$ & \\
\hline \multirow[t]{2}{*}{ Etilismo } & Sim & 30 & $85,7 \%$ & 6 & $6,6 \%$ & $0,000^{* *}$ \\
\hline & Não & 5 & $14,3 \%$ & 85 & $93,4 \%$ & \\
\hline \multirow[t]{2}{*}{ Uso de drogas } & Sim & 1 & $2,9 \%$ & 0 & $0,0 \%$ & $0,105^{*}$ \\
\hline & Não & 34 & $97,1 \%$ & 91 & $100,0 \%$ & \\
\hline \multirow{5}{*}{$\begin{array}{l}\text { Lesão e/ou } \\
\text { alteração na } \\
\text { mucosa oral }\end{array}$} & Sim & 20 & $57,1 \%$ & 42 & $46,2 \%$ & $0,269 * *$ \\
\hline & Não & 15 & $42,9 \%$ & 49 & $53,8 \%$ & \\
\hline & $\begin{array}{l}\text { Varicosidades } \\
\text { linguais }\end{array}$ & 11 & $31,4 \%$ & 15 & $16,5 \%$ & \\
\hline & Estomatite & 1 & $2,9 \%$ & 13 & $14,3 \%$ & \\
\hline & & & & & & $0,115^{*}$ \\
\hline \multirow{2}{*}{ Tipo de lesão } & $\begin{array}{l}\text { Hiperplasia } \\
\text { Fibrosa } \\
\text { Inflamatória }\end{array}$ & 2 & $5,7 \%$ & 5 & $5,5 \%$ & \\
\hline & Outras & 6 & $17,1 \%$ & 9 & $6,6 \%$ & \\
\hline \multirow[t]{2}{*}{ Uso de prótese } & Sim & 32 & $91,4 \%$ & 37 & $40,7 \%$ & $0,000^{*}$ \\
\hline & Não & 3 & $8,6 \%$ & 54 & $59,3 \%$ & \\
\hline \multirow{2}{*}{$\begin{array}{l}\text { Necessidade de } \\
\text { prótese }\end{array}$} & Sim & 33 & $94,3 \%$ & 50 & $54,9 \%$ & $0,000^{*}$ \\
\hline & Não & 2 & $5,7 \%$ & 41 & $45,1 \%$ & \\
\hline
\end{tabular}

* Comparações mediante teste Exato de Fisher; ** Comparações mediante teste Qui-Quadrado; ? :=5\%. 
Tabela 2. Pacientes (frequências e valores percentuais) de acordo com a presença de lesão na mucosa oral para as variáveis de interesse, com as respectivas Odds Ratio (OR) e Intervalos de Confiança (IC) de 2009 a 2012. João Pessoa, PB, 2014.

\begin{tabular}{|c|c|c|c|c|c|c|c|c|}
\hline \multirow[t]{3}{*}{ Variáveis } & \multirow[t]{3}{*}{ Categorias } & \multicolumn{4}{|c|}{ Lesão e/ou alteração na mucosa oral } & \multirow[t]{3}{*}{ OR } & \multirow[t]{3}{*}{ IC (95\%) } & \multirow[t]{3}{*}{ Significância } \\
\hline & & Sim & $\%$ & Não & $\%$ & & & \\
\hline & & $n=62$ & & $n=64$ & & & & \\
\hline \multirow[t]{2}{*}{ Gênero } & Masculino & 20 & $32,2 \%$ & 15 & $23,4 \%$ & - & - & $0,269 * *$ \\
\hline & Feminino & 42 & $67,7 \%$ & 49 & $76,6 \%$ & & & \\
\hline \multirow[t]{2}{*}{ Etnia } & Branco & 45 & $72,6 \%$ & 32 & $50,0 \%$ & 1,452 & $1,087-1,938$ & $0,009 * *$ \\
\hline & Não-Branco & 17 & $27,4 \%$ & 32 & $50,0 \%$ & & & \\
\hline \multirow{2}{*}{$\begin{array}{l}\text { Renda } \\
\text { familiar }\end{array}$} & Até 2 SM's & 48 & $77,4 \%$ & 33 & $51,6 \%$ & 1,501 & $1,143-1,973$ & $0,002^{* *}$ \\
\hline & $\begin{array}{l}3 \text { ou mais } \\
\text { SM's }\end{array}$ & 14 & $22,6 \%$ & 31 & $48,4 \%$ & & & \\
\hline \multirow{3}{*}{$\begin{array}{l}\text { Cidade de } \\
\text { origem }\end{array}$} & Paraíba & 54 & $87,1 \%$ & 47 & $73,4 \%$ & 1,186 & $0,995-1,414$ & $0,055^{* *}$ \\
\hline & Outro & 8 & $12,9 \%$ & 17 & $26,6 \%$ & & & \\
\hline & Estado & & & & & & & \\
\hline \multirow[t]{2}{*}{ Tabagismo } & Sim & 34 & $54,8 \%$ & 21 & $32,8 \%$ & 1,671 & $1,101-2,536$ & $0,013^{* *}$ \\
\hline & Não & 28 & $45,2 \%$ & 43 & $67,2 \%$ & & & \\
\hline \multirow[t]{2}{*}{ Etilismo } & Sim & 23 & $37,1 \%$ & 13 & $20,3 \%$ & 1,826 & $1,019-3,273$ & $0,037^{* *}$ \\
\hline & Não & 39 & $62,9 \%$ & 51 & $79,7 \%$ & & & \\
\hline \multirow{2}{*}{$\begin{array}{l}\text { Uso de } \\
\text { drogas }\end{array}$} & Sim & 1 & $1,6 \%$ & 0 & $0,0 \%$ & - & - & $0,308^{*}$ \\
\hline & Não & 61 & $98,4 \%$ & 64 & $100,0 \%$ & & & \\
\hline \multirow{2}{*}{$\begin{array}{l}\text { Uso de } \\
\text { prótese }\end{array}$} & Sim & 46 & $74,2 \%$ & 23 & $35,9 \%$ & 2,065 & $1,442-2,955$ & $0,000^{* *}$ \\
\hline & Não & 16 & $25,8 \%$ & 41 & $64,1 \%$ & & & \\
\hline \multirow{2}{*}{$\begin{array}{l}\text { Necessidade } \\
\text { de prótese }\end{array}$} & Sim & 51 & $82,3 \%$ & 32 & $50,0 \%$ & 1,645 & $1,255-2,157$ & $0,000^{* *}$ \\
\hline & Não & 11 & $17,7 \%$ & 32 & $50,0 \%$ & & & \\
\hline
\end{tabular}

*Comparações mediante teste Exato de Fisher; ** Comparações mediante teste Qui-Quadrado; [?=5\%. Não foram verificadas diferenças estatisticamente significantes entre pacientes que tinham e os que não tinham registro de lesão e/ou alteração na mucosa oral para as respectivas médias de idade $(\mathrm{p}=0,762)$ e do índice CPOD $(\mathrm{p}=0,765)$. 
No entanto, alguns estudos mostram que os serviços públicos, como os que são ofertados na clínica-escola, são buscados principalmente por pessoas com baixa renda (menor ou igual a 1 salário mínimo) ${ }^{9,10}$ e advindas de locais em que não encontram com facilidade o acesso aos serviços de saúde, principalmente os especializados, como os que podem ser encontrados nas disciplinas de Estomatologia e Prótese Dentária, possível razão pela qual se verificou um maior acesso ao serviço por pacientes oriundos de alguma cidade do interior do Estado da Paraíba.

Ao avaliar a população em estudo, dividindo-a por gênero é possível identificar algumas diferenças estatisticamente significantes $\quad(\mathrm{p} \leq 0,05) \quad$ quanto ao comportamento de algumas variáveis de interesse, como à "etnia", não sendo observado nenhum paciente do gênero masculino declarado não-branco, frente a 49 pacientes do gênero feminino enquadrados nessa categoria, o mesmo ocorrendo para a variável "renda familiar", com nenhum paciente observado do gênero masculino nas subcategorias " 3 e 4 ou mais salários mínimos", frente a 22 e 23 do gênero feminino nas respectivas categorias. Cardoso et al.10 verificaram que, em uma população de Manaus, a renda per capita entre idosos foi maior no gênero masculino, não concordando, portanto, com os resultados do estudo aqui apresentado.

0 conhecimento do histórico dental da população que é atendida em um centro de referência como a clínica-escola, é de fundamental importância para que se tenha um levantamento de dados que permitam entender como se comporta a demanda pelas diferentes áreas da Odontologia para a faixa etária estudada, o que facilita o planejamento do atendimento a ser ofertado. Dessa forma, os dados observados nesse estudo, revelam uma população de maiores de 60 anos com índice CPOD considerado muito alto $(26,0 \pm 5,6)^{11}$ e semelhante ao encontrado por Cardoso et al. $(29,0 \pm 4,3)^{10}$. No presente estudo não foram encontradas diferenças estatisticamente significantes entre os gêneros para as médias do índice CPOD, já Cardoso et al. ${ }^{10}$ observaram essa diferença, sendo a média do índice maior para o gênero masculino.

Observa-se ainda que $65,9 \%$ dos pacientes do presente estudo necessitam de algum tipo de prótese, como forma de substituição de elementos dentários perdidos ao longo da vida. Tal achado é comum em estudos envolvendo idosos, como no estudo de Ribeiro, Veloso e Souza ${ }^{12}$, em que o edentulismo total esteve presente em 63,6\% dos idosos e a necessidade de prótese em $78 \%$ e no de Moura et al. 7 , sendo o edentulismo total verificado em $61,5 \%$ e a necessidade de prótese em 46,9\% dos idosos avaliados. Esses dados refletem tanto o escasso acesso, quanto as formas de intervenção durante um tratamento odontológico a que ocorriam a alguns anos, havendo priorização de exodontias às intervenções preventivas e restauradoras ${ }^{13}$.

Ainda para o uso e a necessidade de próteses dentárias, no presente estudo, os gêneros distribuíram-se de maneira diferente, observando-se que $91,4 \%$ do gênero masculino fazendo uso de prótese; no entanto, observa-se que $94,2 \%$ dos usuários do gênero masculino ainda necessitam de algum tipo de elemento protético. Cardoso et al. ${ }^{10}$ também verificaram haver diferença entre os gêneros quanto ao uso/necessidade de prótese, no entanto, identificaram o gênero feminino como o que mais faz uso e o que mais necessita, tanto de próteses para o arco superior quanto para o inferior.

Para Miotto, Barcellos e Velten ${ }^{14}$, a ausência de dentes e a necessidade de próteses são os fatores mais impactantes na qualidade de vida e a percepção do impacto provocado por problemas bucais, que é maior em indivíduos com mais de 40 anos de idade, sendo a OR (Odds Ratio) para o impacto de 2,77 para a necessidade declarada de prótese parcial removível e de 2,29 para a prótese total removível. Melo et al. ${ }^{15}$ indicam que essa configuração está mudando com o passar dos anos em razão de a população adulta atual estar inserida em um outro momento na 
assistência à saúde, com a descentralização e a oferta de serviços especializados, com melhor qualidade de acesso aos usuários.

Os hábitos adquiridos ao longo da vida, principalmente o de consumo de derivados do tabaco, acompanham a população idosa contemporânea há muito tempo e é reconhecido como um dos principais problemas de saúde pública entre idosos ${ }^{16}$, sendo considerado um hábito deletério à saúde dos tecidos orais ${ }^{17}$. Além disso, esse hábito é de difícil controle nessa faixa etária, tendo em vista os graus de dependência que os derivados do tabaco são capazes de promover no organismo ${ }^{18,19}$. Na presente pesquisa, tanto o "tabagismo" quanto o "etilismo", distribuíram-se de forma estatisticamente diferente entre os gêneros $(p<0,05)$, sendo ainda considerados fatores de risco ao desenvolvimento de lesões orais, com OR de 1,67 e 1,82, respectivamente, o que corresponde aos valores encontrados por Santrain et al. ${ }^{17}$ com valores de OR de 1,7 para fumantes e de 1,6 para o uso do álcool, em associação para as lesões orais em idosos do nordeste do Brasil.

As lesões e/ou alterações em mucosa oral são comumente encontradas em idosos $5,9,17$. No presente estudo, $74,2 \%$ dessas lesões estiveram associadas ao uso de prótese, com uma chance 2,06 vezes maior de desenvolvimento que os não usuários de prótese. Santrain et al. ${ }^{17}$ encontraram que $40 \%$ das lesões orais verificadas em idosos (estomatites, hiperplasias fibrosas, úlceras traumáticas, proliferações em câmara de sucção) estavam presentes em usuários de prótese e Santrain et al. ${ }^{9}$ também encontraram a associação entre a presença de lesões na mucosa oral e o uso de próteses $(\mathrm{p}=0,039)$ em estudo realizado em uma comunidade no nordeste do Brasil, com as lesões em mucosa localizadas principalmente no palato $(67,3 \%)$ e na gengiva $(14,8 \%)$. Em estudo realizado na Universidade de São Paulo com 2250 biópsias, Corrêa et al. ${ }^{5}$ verificaram que $35,01 \%$ das lesões não-malignas eram alterações inflamatórias na mucosa oral, sendo destas
65,34\% hiperplasias fibrosas inflamatórias; e apenas $0,72 \%$ eram desordens vasculares. Da mesma forma, em uma análise de 534 biópsias de pacientes brasileiros realizada por Carvalho et al. ${ }^{8}$ verificou-se que uma das 5 principais lesões diagnosticadas foi a hiperplasia fibrosa com $28,9 \%$ das lesões benignas encontradas, sendo a principal localização a mucosa gengival alveolar $(19,1 \%)$.

Os resultados desse estudo revelam as condições de saúde, bem como as necessidades de cuidado requeridas pelos idosos que procuram o serviço de Odontologia do Centro Universitário, sendo necessário voltar o olhar a essa demanda, bem como às peculiaridades que envolvem o atendimento ao idoso, no intuito de atendê-lo de forma integral, visando proporcionar um aumento na qualidade de vida para os usuários dessa faixa etária.

\section{CONCLUSÃO}

Conclui-se que os pacientes idosos atendidos na clínica-escola de Odontologia do Centro Universitário de João Pessoa são principalmente do gênero feminino, declarados brancos, com renda mensal de até 2 salários mínimos e residentes na Paraíba; ainda, tabagistas, com presença de lesões/alterações na mucosa oral, além de alto índice CPOD e elevada necessidade de uso de prótese dentária.

\section{REFERÊNCIAS}

1. Omran AR. The Epidemiologic Transition: A Theory of the Epidemiology of Population Change. Milbank Memorial Fund Quarterly 2005; 4:731-57.

2. Ministério da Saúde (Br). ONU registra aumento da expectativa de vida no Brasil. 2014. Disponível em: $<$ http://portalsaude.saude.gov.br/index.php/cidadao /principal/agencia-saude/13999-onu-registraaumento-da-expectativa-de-vida-no-brasil >. Acesso em: 29.07.2014.

3. Henrique PR, Bazaga Júnior M, Araújo VC, Junqueira JLC, Furuse C. Prevalência de alterações da mucosa bucal em indivíduos adultos da população de Uberaba, Minas Gerais. RGO 2009; 57(3):261-7.

4. Gil AC. Métodos e técnicas de pesquisa social. 6 ed. São Paulo: Atlas, 2006.

5. Corrêa L, Frigerio MLMA, Sousa SCOM, Novelli MD. Oral lesions in elderly population: a biopsy survey 
using 2250 histopathological records. Gerodontology 2006; 23(1):48-54.

6. Costa EHM, Saintrain MVL, Vieira APGF. Autopercepção da condição de saúde bucal em idosos institucionalizados e não institucionalizados. Ciênc Saúde Coletiva 2010; 15(6):2925-30.

7. Moura C, Cavalcante FC, Catão MHCV, Gusmão ES, Soares RSC, Santillo PMH. Fatores relacionados ao impacto das condições de saúde bucal na vida diária de idosos, Campina Grande, Paraíba, Brasil. Pesquisa Brasileira em Odontopediatria e Clínica Integrada 2011; 11(4):553-9.

8. Carvalho MD, Iglesias DPP, Nascimento GJF, Sobral APV. Epidemiological study of 534 biopsies of oral mucosal lesions in elderly Brazilian patients. Gerodontology 2011; 28(2):111-5.

9. Santrain MVL, Almeida CB, Naruse TMO, Gonçalves VP. Oral lesions in elderly patients of a comunity in Brazilian Northeast. Gerontology 2013; 30(4):283-7. 10. Cardoso EM, Parente RCP, Vettore MV, Rebello MAB. Condição de saúde bucal em idosos residentes no município de Manaus, Amazonas: estimativas por sexo. Rev Bras Epidemiol 2011; 14(1):131-40.

11. Ministério da Saúde (Br). Projeto SB Brasil 2010. Disponível

em: <http://bvsms.saude.gov.br/bvs/publicacoes/pesqui sa_nacional_saude_bucal.pdf $>$. Acesso em: 12.11.2013. 12. Ribeiro ILA, Veloso HHP, Souza KC. Caracterização da saúde bucal de idosos em uma instituição beneficente de longa permanência de João Pessoa-PB, Brasil. Revista Cubana de Estomatología 2012; 49(3):193-203.
13. Pinheiro RS, Torres TZG. Access to oral health services between Brazilian States. Ciênc Saúde Coletiva. 2006; 11:999-1010.

14. Miotto MHMB, Barcellos LA, Velten DB. Cienc Saúde Coletiva. 2012; 17(2):397-407.

15. Melo ALSF, Andrade SR, Moysés SJ, Erdmann AL. Saúde bucal na rede de atenção e processo de regionalização. Ciênc Saúde Coletiva. 2013; 18(1):205-14.

16. Marinho V, Laks J, Coutinho ESF, Blay SL. Tobacco use among the elderly: a systematic review and metaanalysis Cad. Saúde Pública 2010; 26(12):2213-33.

17. Santrain MVL, Holanda TG, Bezerra TMM, Almeida P. Prevalence of soft tissue oral lesion in elderly and its relations with deleterious habits. Gerodontology 2012; 29:130-4.

18. Yildiz D. Nicotine, its metabolism and an overview of its biological effects. Toxicon 2004; 43:619-32.

19. Ribeiro ILA, Veloso HHP. Influência do Tabagismo nas alterações pulpares. ROBRAC 2012; 21(58):5705.

CONTRIBUIÇÕES
Isabella Lima Arrais Ribeiro foi responsável pela
concepção, análise e interpretação dos dados e,
redação do artigo. Eduardo Dias Ribeiro atuou na
concepção e revisão crítica. Waldemir Gonçalves
de Abrantes responsabilizou-se pelo delineamento
e redação do artigo. Celso Koogi Sonoda
desenvolveu a revisão crítica do artigo.

\section{Como citar este artigo (Vancouver):}

Ribeiro ILA, Ribeiro ED, Abrantes WG, Sonoda CK. Caracterização socioeconômica e condição de saúde oral de idosos atendidos em uma clínica-escola de odontologia. REFACS [Internet]. 2016 [citado em (inserir dia, mês e ano de acesso)]; 4(3):177-184. Disponível em: (link de acesso). DOI: $10.18554 /$ refacs.v4i3.1765.

Como citar este artigo (ABNT):

RIBEIRO, I.L.A.; RIBEIRO, E.D.; ABRANTES, W.G.; SONODA, C.K. Caracterização socioeconômica e condição de saúde oral de idosos atendidos em uma clínica-escola de odontologia. REFACS, Uberaba, MG, v. 4, n. 3, p. 177-184, 2016. Disponível em: (link de acesso). Acesso em: (inserir dia, mês e ano de acesso). DOI: 10.18554 /refacs.v4i3.1765.

Como citar este artigo (APA):

Ribeiro I.L.A, Ribeiro E.D, Abrantes W.G \& Sonoda C.K. (2016). Caracterização socioeconômica e condição de saúde oral de idosos atendidos em uma clínica-escola de odontologia. REFACS, 4(3), 177-184. Recuperado em (inserir dia, mês e ano de acesso) (inserir link de acesso). DOI: 10.18554/refacs.v4i3.1765. 